\title{
An Intelligent Algorithm for Skin Cancer Detection
}

\author{
Amin Nazerzadeh1, Afsaneh Nouri Houshyar ${ }^{2 *}$, Alireza Jahed ${ }^{3}$ \\ ${ }^{1}$ UCI Division of Continuing Education, University of California Irvine, Irvine, CA, USA \\ ${ }^{2}$ School of Engineering, California State Polytechnic University, Panoma, CA, USA \\ ${ }^{3}$ School of Business, Khatam University, Tehran, Iran \\ Email: ^afsanehn@cpp.edu
}

How to cite this paper: Nazerzadeh, A., Houshyar, A.N. and Jahed, A. (2020) An Intelligent Algorithm for Skin Cancer Detection. Intelligent Control and Automation, 11, 25-31.

https://doi.org/10.4236/ica.2020.111003

Received: November 20, 2019

Accepted: February 25, 2020

Published: February 28, 2020

Copyright (c) 2020 by author(s) and Scientific Research Publishing Inc. This work is licensed under the Creative Commons Attribution International License (CC BY 4.0).

http://creativecommons.org/licenses/by/4.0/

\begin{abstract}
Nowadays, computer vision as an interdisciplinary field is growing in different areas such as medical, electronics, etc. In the field, detection and particularly image segmentation is an essential task in which is difficult to find the appropriate one based on the application. In this paper, a new algorithm is proposed to segment the lesion from background. The algorithm is based on log edge detector with iterative median filtering. We have tested our algorithm on 20 dermoscopic images and compare the lesion detection results with those manually segmented by dermatologists. The experiments represent the effectiveness of proposed algorithm.
\end{abstract}

\section{Keywords}

Skin Cancer, Malignant, Benign, Image Processing, Log Edge Detector, Segmentation

\section{Introduction}

Skin cancer is determined by the abnormal and uncontrolled spread of cells which make up the skin. It is classified as benign or malignant. Malignant melanoma as a deadliest skin cancer tumor has a probability of metastasis and grows rapidly to other organs. Thus, the quick detection of melanoma is of extreme importance [1]. Investigations have demonstrated that the cure rate is approximately $100 \%$ if it is recognized and treated early [2]. For this reason, the automatic or semi-automatic interpretation of results became unavoidable [1].

Advances in clinical dermoscopy have considerably contributed to improve diagnosis and reduce risks. It entrusts $10 \%-27 \%$ higher sensitivity to diagnosing melanoma by dermatologists [3] [4]. Hence, development of dermoscopic diag- 
nosis still increasing in subject over the last years. Different methods have been developed and tested on a wide range of tasks to assist the system designer to choose the most appropriate approach. Clinical outcome of melanoma can potentially be ameliorated by increasing the accuracy of tumors localization during the treatment. Hence, the risk of mortality caused by late diagnosis can be reduced if the set-up uncertainties are minimized. One of the particular uncertainties in the system is determining the borders between tissues to find the features for accurate detection. The border detection is an important task because it provides the basis of extracting the features of lesion such as size and symmetry axes, and most conversant dermoscopic features, such as radial streaming and pseudopods. Since the transition between skin and lesion is almost of low contrast, and memberships sin lesion boundary are intrinsically fuzzy, the exact determination of borders is a difficult task [1] [5] [6] [7].

In this study, we place the concentrate on determining the lesion borders. Our approach is particularly appealing and differs from others as it is robust in presence of hair and noise. Our motivation to perform segmentation of skin cancer images has been to facilitate the robust algorithm for automatic tracking of melanoma in detection systems which can delineate the lesions at risk.

Experiments and comparison results of more than 30 skin lesion images illustrate that the proposed algorithm results are very close to borders traced manually by a dermatologist, even for the images with high asymmetric lesions, weak edges and artifacts.

The remainder of the article is organized as follows: a short review on previous studies of skin cancer segmentation is presented. Then, the proposed algorithm for segmenting the melanoma from the images is explained in detail. Third, the experimental results are carried out on RGB images to evaluate the validity of our algorithm. The results are compared with the traditional algorithm to demonstrate the superiority of proposed algorithm. Finally, the article is concluded with discussion.

\section{Previous Studies}

In the past, several approaches have been proposed for segmentation of skin lesions in automatic diagnosis of malignant and benign. F. Xie, A. Bovik, 2013, proposed an algorithm to combine genetic algorithm (GA) and a self-generating neural network (SGNN). They feed SGNN using optimal samples selected by GA algorithm which optimized and stabilized the clustering outcome. The overall model leads to automatic segmentation without any manual interaction. They compared the model with other segmentation methods and got more accurate results [5]. A.T. Beuren et al., 2012, presented a new approach for extraction of lesions region based on morphological operators and binarization technique. They concluded that their proposed algorithms can effectively detect the lesions [8]. H. Wang, 2011, developed a watershed segmentation algorithm by utilizing the neural network classifier to reduce to error rates. They could achieve 
the error rate of $11.09 \%$ in compare to their previous work [9]. H. Zhou et al., 2011, introduced a new segmentation method based on standard GVF algorithm. They applied mean shift operation within the standard GVF cost function and presented accurate results [10]. In another study [11], the improved fuzzy clustering algorithms and its application in brain image segmentation was investigated and they concluded that their algorithm could accurately segment brain tissue.

M. Silveira et al. reviewed different methods of skin lesion segmentation and compared the different methods in three type of thresholding, edge-based, and region-based [12]. X. Yuan, 2008, presented a segmentation algorithm based on evolution strategy. In this paper, they considered the segmentation problem as numerical optimization problem and applied ES as a search algorithm and designed an objective function for its application. The histogram-based consistency verification rule was used for automatic refinement of results. Experiments showed the robust application of algorithm which its efficiency doesn't depend the initial values [13]. L. Xiao et al., 2019, proposed a method to improve the performance of traditional Otsu method. They utilized 2D histogram projection analysis for threshold segmentation based on set mapping and trapezoid region intercept histogram. The results demonstrate the better performance and could achieve a balance between the noise elimination and the detail retention in image segmentation [14]. Ganster et al, 2001, proposed a system for melanoma detection. One of purposes was to develop the segmentation of lesion in skin cancer images. They performed fusion of thresholding and color clustering algorithms and could achieve impressive results [15].

\section{Proposed Methodology}

As mentioned before, lots of skin cancer segmentation algorithms have been presented in the literature to help dermatologists in accurate detection of melanoma. However, the noise and hairs affect significantly on segmentation precision and also the main purpose is to achieve the algorithm which segments the lesions in either malignant or benign lesion images.

Motivated by these issues, in this paper we propose an algorithm for skin lesion segmentation which has the good performance even in presence of hair and noise. First of all, since in this process, the high-level variations between intensities of the image are important, it is beneficial to convert the image into greyscale, in which the minimum intensity value was equal to 0 , and the maximum intensity value was equal to 255 . Secondly, the image is filtered through computing the first order derivative of Gaussian and computing the magnitude and orientation of gradient vector. Based on the experiment, the threshold value is determined through considering the top $40 \%$ highest gradient pixels and the binary image is obtained. Lastly, The XOR of each pixel with the next pixel is performed to determine the boundaries of the image. The proposed algorithm of skin lesions segmentation is shows in Algorithm 1. 


\section{Results and Discussion}

In this section, the results are discussed, and the validity of the proposed algorithm is investigated. To quantitatively evaluate the performance of the algorithm, experiments are carried out on thirty RGB images which have been segmented manually by a dermatologist. These manual segmentations are treated as ground truth. These images have been collected from the Cancer Council NSW and other referenced papers. The obtained images will then be compared with the ones from Log edge detector algorithm to demonstrate its superiority. Figure 1 shows a sample of a ground truth image.

We validate our algorithm by comparing its statistical results with the ground truth as well as the results from the Log edge detector. Figure 2 shows the sample border detection results. The proposed algorithm demonstrates effective border lines.

Algorithm 1. The proposed algorithm of skin lesions segmentation.

\section{Input: RGB image \\ Output: Lesion Segmentation}

1) The RGB image is converted to grayscale image

2) The gradient of pixels in an image are calculated using the first order derivative of Gaussian.

3) Compute magnitude and orientation of gradient vector

4) The top $\mathrm{p} \%$ highest gradient pixels are considered as threshold value.

5) The image is converted into black and white image, based on threshold

6) XOR of each pixel with the next pixel in an image is created which shows the edge of image.

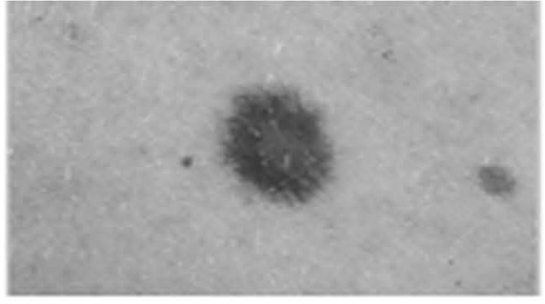

(a)

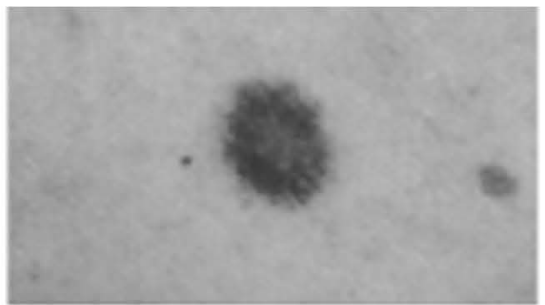

(b)

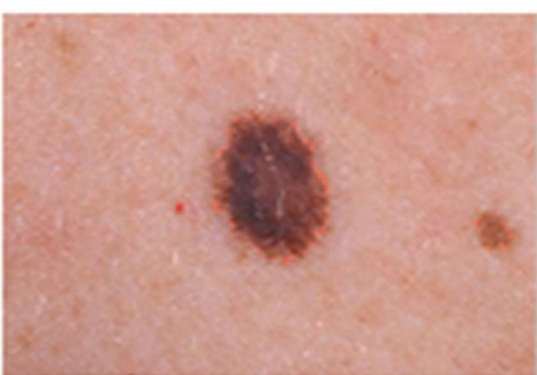

Figure 1. Ground truth image.

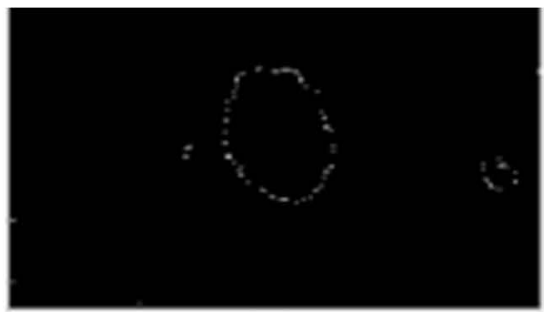

(c)

Figure 2. (a) Grayscale Image, (b) gaussian filter and XOR of each pixel with the next pixel, (c) Laplacian of gradient (Log) edge detector. 
Figure 3 shows the segmented results using our proposed algorithm on three more images including benign and malignant lesions.

The images are polluted with the additive noise of level $5 \%(\mathrm{SNR}=26 \mathrm{~dB})$ to make higher noisy image and to evaluate the performance of the proposed algorithm. Figure 4 shows the results of segmented image showed earlier in Figure 2.

Additionally, to validate the algorithm, the Border Error (BE) and SNR between the proposed algorithm and Log edge detector algorithm are computed. The percentage border error is computed as,

$$
\text { Border Error }=\frac{\text { Area }(\text { Automatic Border } \oplus \text { Manual Border })}{\text { Area }(\text { Manual Border })} \times 100 \%
$$

and the $S N R$ is computed as,

$$
S N R=10 \log \left(P_{\text {image }} / P_{\text {Noise }}\right)
$$

Table 1 shows the computed results for our proposed algorithm and Log edge detector algorithm in simple and noisy conditions.

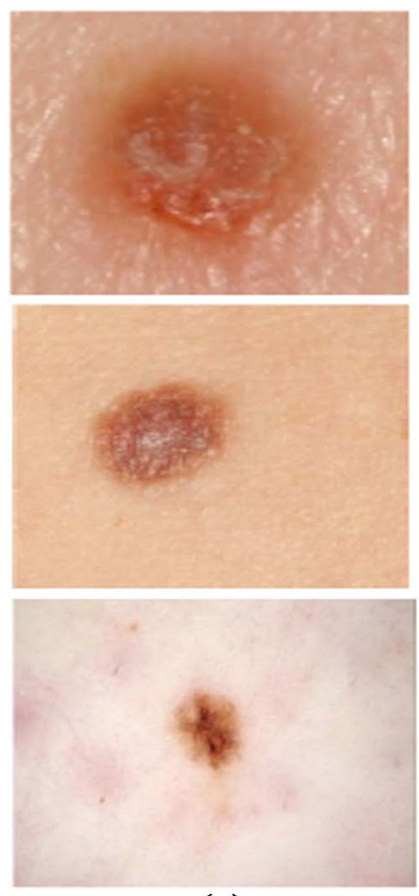

(a)
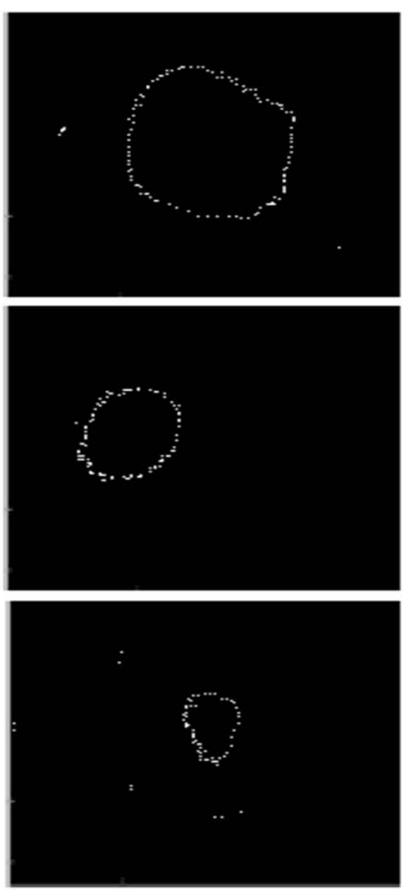

(b)

Figure 3. (a) Original images, (b) Segmented images using the proposed algorithm.

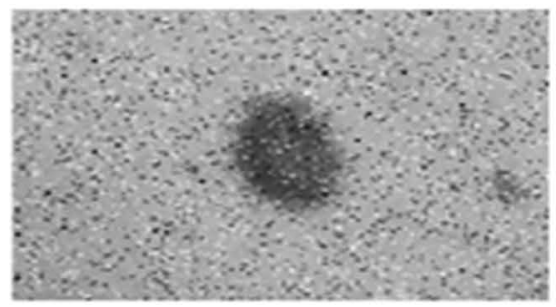

(a)

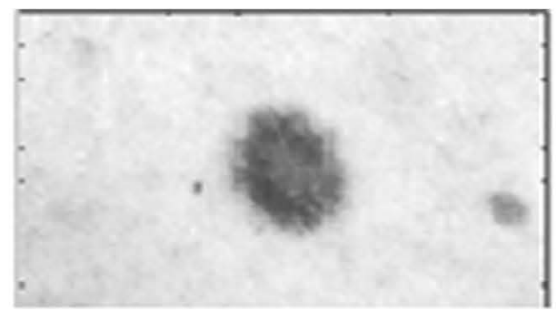

(b)

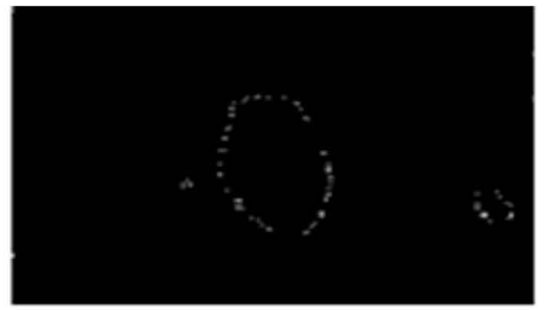

(c)

Figure 4. (a) Noisy image with $\mathrm{SNR}=26 \mathrm{~dB}$, (b) grayscale Image with additive noise, (c) Laplacian of gradient (Log) edge detector. 
Table 1. Comparison of BE and SNR between our proposed algorithm and Log edge detector algorithm in Normal noise level, additive noise of $2 \%$ and $5 \%$.

\begin{tabular}{ccc}
\hline & Proposed algorithm & Log edge detector algorithm \\
\hline BE-Normal noise level & 0.22 & 0.25 \\
BE-additive noise of level 2\% & 0.32 & 0.32 \\
BE-additive noise of level 5\% & 0.39 & 0.42 \\
SNR-Normal noise level & -16.07 & -17.01 \\
SNR-additive noise of level 2\% & -18.23 & -18.95 \\
SNR-additive noise of level 5\% & -20.11 & -22.03 \\
\hline
\end{tabular}

The experimental results demonstrate that proposed algorithm performs better than Log edge detector.

\section{Conclusion}

In this paper, we presented a generic framework for automatic skin lesion segmentation based on Log edge detection algorithm. Experiments were performed on 30 images with known ground truth obtained by manual detection of borders by a dermatologist. Our results show that the proposed algorithm is more efficient in detecting the edges than Log edge detection algorithm. The experiments indicate that the obtained results from our proposed algorithm are very close to that obtained by human.

\section{Conflicts of Interest}

The authors declare no conflicts of interest regarding the publication of this paper.

\section{References}

[1] Dorrell, D.N. and Strowd, L.C. (2019) Skin Cancer Detection Technology. Dermatologic Clinics, 37, 527-536. https://doi.org/10.1016/j.det.2019.05.010

[2] Di Leo, G., Liguori, C., Paolillo, A. and Sommella, P. (2008) An Improved Procedure for the Automatic Detection of Dermoscopy Structures in Digital ELM Images of Skin Lesions. In: Proceedings of the IEEE International Conference on Virtual Environment, Human-Computer Interfaces, and Measurement Systems, Istanbul, Turkey. https://doi.org/10.1109/VECIMS.2008.4592778

[3] Shakourian Ghalejoogh, G., Montazery Kordy, H. and Ebrahimi, F. (2020) A Hierarchical Structure Based on Stacking Approach for Skin Lesion Classification. EXpert Systems with Applications, 1451, Article No. 113127. https://doi.org/10.1016/j.eswa.2019.113127

[4] Garbe, C., Peris, K., Hauschild, A., Saiag, P., Middleton, M., Spatz, A., Grob, J.J., Malvehy, J., Bishop, J.N., Stratigos, A., Pehamberger, H. and Eggermont, A.M. (2012) Diagnosis and Treatment of Melanoma. European Consensus-Based Interdisciplinary Guideline. European Journal of Cancer, 48, 2375-2390. https://doi.org/10.1016/j.ejca.2012.06.013

[5] Xie, F. and Bovik, A. (2013) Automatic Segmentation of Dermoscopy Images Using 
Self-Generating Neural Networks Seeded by Genetic Algorithm. Pattern Recognition, 46, 1012-1019. https://doi.org/10.1016/j.patcog.2012.08.012

[6] Pereira, P.M.M., Fonseca-Pinto, R., Pedro Paiv, R., Assuncao, P.A.A., Tavor, L.M.N., Thomaz, L.A. and Faria, S.M.M. (2020) Skin Lesion Classification Enhancement Using Border-Line Features-The Melanoma vs Nevus Problem. Biomedical Signal Processing and Control, 57, Article No. 101765. https://doi.org/10.1016/j.bspc.2019.101765

[7] Celebi, M.E., Iyatomi, H., Schaefer, G. and Stoecker, W.V. (2009) Lesion Border Detection in Dermoscopy Images. Computerized Medical Imaging and Graphics, 33, 148-153. https://doi.org/10.1016/j.compmedimag.2008.11.002

[8] Beuren, A.T., Janasieivicz, R., Grando, N. and Facon, J. (2012) Skin Melanoma Segmentation by Morphological Approach. In: Proceedings of the International Conference on Advances in Computing, Communications and Informatics, ACM, New York. https://doi.org/10.1145/2345396.2345553

[9] Wang, H., Moss, R.H., Chen, X., Stanley, R.J., Stoecker, W.V., Celebi, M.E., Malters, J.M., Grichnik, J.M., Marghoob, A.A., Rabinovitz, H.S., Menzies, S. and Szalapski, T.M. (2011) Modified Watershed Technique and Post-Processing for Segmentation of Skin Lesions in Dermoscopy Images. Computerized Medical Imaging and Graphics, 35, 116-120. https://doi.org/10.1016/j.compmedimag.2010.09.006

[10] Zhou, H., Schaefer, G., Emre Celebi, M., Lin, F. and Liu, T. (2011) Gradient Vector Flow with Mean Shift for Skin Lesion Segmentation. Computerized Medical Imaging and Graphics, 35, 121-127. https://doi.org/10.1016/j.compmedimag.2010.08.002

[11] Ren, T.B., Wang, H.H., Feng, H.L., Xu, C.S., Liu, G.S. and Ding, P. (2019) Study on the Improved Fuzzy Clustering Algorithm and Its Application in Brain Image Segmentation. Applied Soft Computing, 81, Article No. 105503. https://doi.org/10.1016/j.asoc.2019.105503

[12] Silveira, M., Nascimento, J.C., Marques, J.S., Marçal, A.R.S., Mendonça, T., Yamauchi, S., Maeda, J. and Rozeira, J. (2009) Comparison of Segmentation Methods for Melanoma Diagnosis in Dermoscopy Images. IEEE Journal of Selected Topics in Signal Processing, 3, 35-45. https://doi.org/10.1109/JSTSP.2008.2011119

[13] Yuan, X., Situ, N. and Zouridakis, G. (2008) Automatic Segmentation of Skin Lesion Images Using Evolution Strategies. Biomedical Signal Processing and Control, 3 , 220-228. https://doi.org/10.1016/j.bspc.2008.02.003

[14] Xiao, L., Ouyang, H.L. and Fan, C.D. (2019) An Improved Otsu Method for Threshold Segmentation Based on Set Mapping and Trapezoid Region Intercept Histogram. Optik, 196, Article No. 163106. https://doi.org/10.1016/j.ijleo.2019.163106

[15] Ganster, H., Pinz, P., Rohrer, R., Wildling, E., Binder, M. and Kittler, H. (2001) Automated Melanoma Recognition. IEEE Transactions on Medical Imaging, 20, 233-239. https://doi.org/10.1109/42.918473 